\title{
Catching Element Formation In The Act The Case for a New MeV Gamma-Ray Mission: Radionuclide Astronomy in the 2020s
}

\author{
A White Paper for the 2020 Decadal Survey
}

\author{
Authors \\ Chris L. Fryer, Los Alamos National Laboratory, fryer@lanl.gov, (505) 665-3394 \\ Frank Timmes, Arizona State University, fxtimmes@ gmail.com, (480) 965-4274 \\ Aimee L. Hungerford, Los Alamos National Laboratory \\ Aaron Couture, Los Alamos National Laboratory
}

Fred Adams, University of Michigan

Wako Aoki, National Astronomical Observatory of Japan

Almudena Arcones, Technische Universität Darmstadt

David Arnett, University of Arizona

Katie Auchettl, DARK, Niels Bohr Institute, University of Copenhagen

Melina Avila, Argonne National Laboratory

Carles Badenes, University of Pittsburgh

Eddie Baron, University of Oklahoma

Andreas Bauswein, GSI Helmholtzzentrum für Schwerionenforschung

John Beacom, Ohio State University

Jeff Blackmon, Louisiana State University

Stéphane Blondin, CNRS \& Pontificia Universidad Catolica de Chile

Peter Bloser, Los Alamos National Laboratory

Steve Boggs, UC San Diego

Alan Boss, Carnegie Institution for Science

Terri Brandt, NASA Goddard Space Flight Center

Eduardo Bravo, Universitat Politècnica de Catalunya

Ed Brown, Michigan State University

Peter Brown, Texas A\&M University

Steve Bruenn, University of Florida Atlantic

Carl Budtz-Jørgensen, Technical University of Denmark

Eric Burns, NASA Goddard Space Flight Center, Universities Space Research Association

Alan Calder, Stony Brook University

Regina Caputo, NASA Goddard Space Flight Center 
Art Champagne, University of North Carolina at Chapel Hill

Roger Chevalier, University of Virginia

Alessandro Chieffi, Istituto Nazionale di Astrofisica

Kelly Chipps, Oak Ridge National Laboratory

David Cinabro, Wayne State University

Ondrea Clarkson, University of Victoria

Don Clayton, Clemson University

Alain Coc, Université Paris

Devin Connolly, TRIUMF

Charlie Conroy, Harvard University

Benoit Côté, Konkoly Observatory

Sean Couch, Michigan State University

Nicolas Dauphas, University of Chicago

Richard James deBoer, University of Notre Dame

Catherine Deibel, Louisiana State University

Pavel Denisenkov, University of Victoria

Steve Desch, Arizona State University

Luc Dessart, Universidad de Chile

Roland Diehl, Max Planck Institute for Extraterrestrial Physics Garching

Carolyn Doherty, Konkoly Observatory

Inma Domínguez, University of Granada

Subo Dong, Kavli Institute for Astronomy and Astrophysics, Peking University

Vikram Dwarkadas, University of Chicago

Doreen Fan, Lawrence Berkeley National Laboratory

Brian Fields, University of Illinois

Carl Fields, Michigan State University

Alex Filippenko, University of California Berkeley

Robert Fisher, University of Massachusetts Dartmouth

Francois Foucart, University of New Hampshire

Claes Fransson, Stockholm University

Carla Fröhlich, North Carolina State University

George Fuller, University of California San Diego

Brad Gibson, University of Hull

Viktoriya Giryanskaya, Princeton University

Joachim Görres, University of Notre Dame

Stéphane Goriely, Université Libre de Bruxelles

Sergei Grebenev, Space Research Institute, Russian Academy of Sciences

Brian Grefenstette, California Institute of Technology

Evan Grohs, Los Alamos National Laboratory

James Guillochon, Harvard-Smithsonian Center for Astrophysics

Alice Harpole, Stony Brook University

Chelsea Harris, Michigan State University

J. Austin Harris, Oak Ridge National Laboratory

Fiona Harrison, California Institute of Technology

Dieter Hartmann, Clemson University 
Masa-aki Hashimoto, Kyushu University

Alexander Heger, Monash University

Margarita Hernanz, Institute of Space Sciences

Falk Herwig, University of Victoria

Raphael Hirschi, Keele University

Raphael William Hix, Oak Ridge National Laboratory

Peter Höflich, Florida State University

Robert Hoffman, Lawrence Livermore National Laboratory

Cole Holcomb, Princeton University

Eric Hsiao, Florida State University

Christian Iliadis, University of North Carolina at Chapel Hill

Agnieszka Janiuk, Center for Theoretical Physics Polish Academy of Sciences

Thomas Janka, Max Planck Institute for Astrophysics

Anders Jerkstrand, Max Planck Institute for Astrophysics

Lucas Johns, University of California San Diego

Samuel Jones, Los Alamos National Laboratory

Jordi José, Universitat Politècnica de Catalunya

Toshitaka Kajino, The University of Tokyo

Amanda Karakas, Monash University

Platon Karpov, University of California Santa Cruz

Dan Kasen, University of California Berkeley

Carolyn Kierans, University of California Berkeley

Marc Kippen, Los Alamos National Laboratory

Oleg Korobkin, Los Alamos National Laboratory

Chiaki Kobayashi, University of Hertfordshire

Cecilia Kozma, Stockholm House of Science

Saha Krot, University of Hawaii

Pawan Kumar, University of Texas at Austin

Irfan Kuvvetli, Technical University of Denmark

Alison Laird, University of York

(John) Martin Laming, Naval Research Laboratory

Josefin Larsson, KTH Royal Institute of Technology

John Lattanzio, Monash University

James Lattimer, Stony Brook University

Mark Leising, Clemson University

Annika Lennarz, TRIUMF

Eric Lentz, University of Tennessee

Marco Limongi, Istituto Nazionale di Astrofisica

Jonas Lippuner, Los Alamos National Laboratory

Eli Livne, Racah Institute of Physics, The Hebrew University

Nicole Lloyd-Ronning, Los Alamos National Laboratory

Richard Longland, North Carolina State University

Laura A. Lopez, Ohio State University

Maria Lugaro, Konkoly Observatory

Alexander Lutovinov, Space Research Institute of the Russian Academy of Science 
Kristin Madsen, California Institute of Technology

Chris Malone, Los Alamos National Laboratory

Francesca Matteucci, Trieste University, INAF, INFN

Julie McEnery, NASA Goddard Space Flight Center

Zach Meisel, Ohio University

Bronson Messer, Oak Ridge National Laboratory

Brian Metzger, Columbia University

Bradley Meyer, Clemson University

Georges Meynet, University Of Geneva

Anthony Mezzacappa, Oak Ridge National Laboratory, University of Tennessee

Jonah Miller, Los Alamos National Laboratory

Richard Miller, Johns Hopkins University Applied Physics Laboratory

Peter Milne, University of Arizona

Wendell Misch, Shanghai Jiao Tong University

Lee Mitchell, Naval Research Laboratory

Philipp Mösta, University of California Berkeley

Yuko Motizuki, RIKEN Nishina Center

Bernhard Müller, Monash University

Matthew Mumpower, Los Alamos National Laboratory

Jeremiah Murphy, Florida State University

Shigehiro Nagataki, RIKEN

Ehud Nakar, Tel Aviv University

Ken'ichi Nomoto, Tokyo University

Peter Nugent, Lawrence Berkeley National Laboratory

Filomena Nunes, Michigan State University

Brian O'Shea, Michigan State University

Uwe Oberlack, Johannes Gutenberg University

Steven Pain, Oak Ridge National Laboratory

Lucas Parker, Los Alamos National Laboratory

Albino Perego, Universitá degli Studi di Milano Bicocca

Marco Pignatari, University of Hull

Gabriel Martínez Pinedo, Technische Universität Darmstadt

Tomasz Plewa, Florida State University

Dovi Poznanski, Tel Aviv University

William Priedhorsky, Los Alamos National Laboratory

Boris Pritychenko, Brookhaven National Laboratory

David Radice, Institute for Advanced Study, Princeton University

Enrico Ramirez-Ruiz, University of California Santa Cruz

Thomas Rauscher, University of Basel and University of Hertfordshire

Sanjay Reddy, Institute for Nuclear Theory, University of Washington

Ernst Rehm, Argonne National Laboratory

Rene Reifarth, Goethe Universität Frankfurt

Debra Richman, Michigan State University, National Superconducting Cyclotron Laboratory

Paul Ricker, University of Illinois

Nabin Rijal, Florida State University, National Superconducting Cyclotron Laboratory 
Luke Roberts, Michigan State University, National Superconducting Cyclotron Laboratory Friedrich Röpke, Universität Heidelberg, Heidelberg Institute for Theoretical Studies

Stephan Rosswog, Stockholm University

Ashley J. Ruiter, University of New South Wales Canberra

Chris Ruiz, TRIUMF

Daniel Wolf Savin, Columbia University

Hendrik Schatz, Michigan State University

Dieter Schneider, Los Alamos National Laboratory

Josiah Schwab, University of California Santa Cruz

Ivo Seitenzahl, University of New South Wales Canberra

Ken Shen, University of California Berkeley

Thomas Siegert, Max Planck Institute for Extraterrestrial Physics Garching

Stuart Sim, Queen's University Belfast

David Smith, University of California Santa Cruz

Karl Smith, Los Alamos National Laboratory

Michael Smith, Oak Ridge National Laboratory

Jesper Sollerman, The Oskar Klein Centre, Department of Astronomy

Trevor Sprouse, University of Notre Dame

Artemis Spyrou, Michigan State University

Sumner Starrfield, Arizona State University

Andrew Steiner, University of Tennessee, Knoxville, Oak Ridge National Laboratory

Andrew W. Strong, Max Planck Institut für Extraterrestrische Physik

Tuguldur Sukhbold, Ohio State University

Nick Suntzeff, Texas A\&M University

Rebecca Surman, University of Notre Dame

Toru Tanimori, Kyoto University

Lih-Sin The, Clemson University

Friedrich-Karl Thielemann, University of Basel and GSI Darmstadt

Alexey Tolstov, Open University of Japan, University of Tokyo

Nozomu Tominaga, Konan University

John Tomsick, University of California Berkeley

Dean Townsley, University of Alabama

Pelagia Tsintari, Central Michigan University

Sergey Tsygankov, University of Turku

David Vartanyan, Princeton University

Tonia Venters, NASA Goddard Space Flight Center

Tom Vestrand, Los Alamos National Laboratory

Jacco Vink, University of Amsterdam

Roni Waldman, Hebrew University

Lifang Wang, Texas A\&M University

Xilu Wang, University of Notre Dame

MacKenzie Warren, Michigan State University

Christopher West, Concordia University

J. Craig Wheeler, University of Texas at Austin

Michael Wiescher, University of Notre Dame 
Christoph Winkler, European Space Agency

Lisa Winter, Los Alamos National Laboratory

Bill Wolf, Arizona State University

Richard Woolf, Naval Research Laboratory

Stan Woosley, University of California Santa Cruz

Jin Wu, Argonne National Laboratory

Chris Wrede, Michigan State University

Shoichi Yamada, Waseda University

Patrick Young, Arizona State University

Remco Zegers, Michigan State University

Michael Zingale, Stony Brook University

Simon Portegies Zwart, Leiden University

Thematic Areas:

PRIMARY: Stars and Stellar Evolution

SECONDARY: Galaxy Evolution

Projects/Programs Emphasized:

1. All-sky Medium Energy Gamma-ray Observatory (AMEGO)

https://asd.gsfc.nasa.gov/amego/

2. e-ASTROGAM

http://eastrogam.iaps.inaf.it

3. Compton Spectrometer and Imager (COSI)

http://cosi.ssl.berkeley.edu

4. Electron-Tracking Compton Camera (ETCC)

5. Lunar Occultation Explorer (LOX) 


\section{Executive Summary}

Gamma-ray astronomy explores the most energetic photons in nature to address some of the most pressing puzzles in contemporary astrophysics. It encompasses a wide range of objects and phenomena: stars, supernovae, novae, neutron stars, stellar-mass black holes, nucleosynthesis, the interstellar medium, cosmic rays and relativistic-particle acceleration, and the evolution of galaxies. $\mathrm{MeV} \gamma$-rays provide a unique probe of nuclear processes in astronomy, directly measuring radioactive decay, nuclear de-excitation, and positron annihilation. The substantial information carried by $\gamma$-ray photons allows us to see deeper into these objects, the bulk of the power is often emitted at $\gamma$-ray energies, and radioactivity provides a natural physical clock that adds unique information.

New science will be driven by time-domain population studies at $\gamma$-ray energies. This science is enabled by next-generation $\gamma$-ray instruments with one to two orders of magnitude better sensitivity, larger sky coverage, and faster cadence than all previous $\gamma$-ray instruments. This transformative capability permits: (a) the accurate identification of the $\gamma$-ray emitting objects and correlations with observations taken at other wavelengths and with other messengers; (b) construction of new $\gamma$-ray maps of the Milky Way and other nearby galaxies where extended regions are distinguished from point sources; and (c) considerable serendipitous science of scarce events - nearby neutron star mergers, for example. Advances in technology push the performance of new $\gamma$-ray instruments to address:

* How do white dwarfs explode as Type Ia Supernovae (SNIa)?

$\star$ What is the distribution of ${ }^{56} \mathrm{Ni}$ production within a large population of SNIa?

$\star$ How do SNIa $\gamma$-ray light curves and spectra correlate with their UV/optical/IR counterparts?

$\star$ How do massive stars explode as core-collapse supernovae?

$\star$ How are newly synthesized elements spread out within the Milky Way Galaxy?

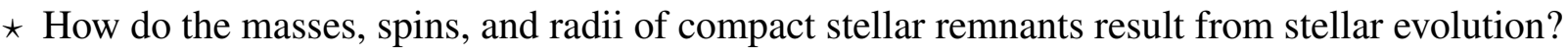

$\star$ How do novae enrich the Galaxy in heavy elements?

* What is the source that drives the morphology of our Galaxy's positron annihilation $\gamma$-rays?

$\star$ How do neutron star mergers make most of the stable r-process isotopes?

Over the next decade, multi-messenger astronomy will probe the rich astrophysics of transient phenomena in the sky, including light curves and spectra from supernovae and interacting binaries, gravitational and electromagnetic signals from the mergers of compact objects, and neutrinos from the Sun, massive stars, and the cosmos. During this new era, the terrestrial Facility for Rare Isotope Beams (FRIB) and Argonne Tandem Linac Accelerator System (ATLAS) will enable unprecedented precision measurements of reaction rates with novel direct and indirect techniques to open perspectives on transient objects such as novae, $\mathrm{x}$-ray bursts, kilonovae, and the rapid neutron capture process. This ongoing explosion of activity in multi-messenger astronomy powers theoretical and computational developments, in particular the evolution of community-driven, open-knowledge software instruments. The unique information provided by $\mathrm{MeV} \gamma$-ray astronomy to help address these frontiers makes now a compelling time for the astronomy community to strongly advocate for a new $\gamma$-ray mission to be operational in the 2020s and beyond. 


\section{Supernovae And Other Cosmic Explosions}

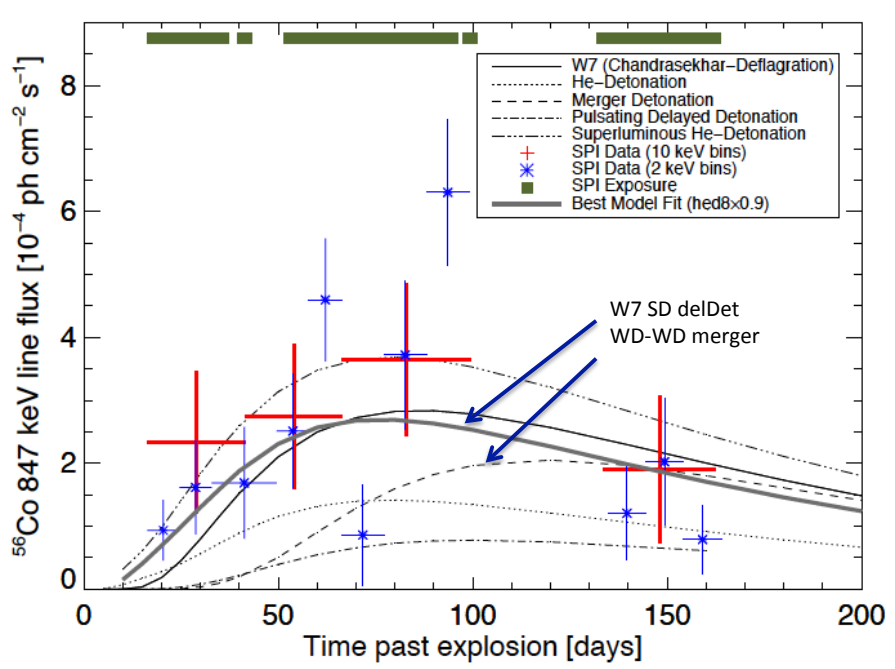

Figure 1: SN2014J was the first SNIa within reach

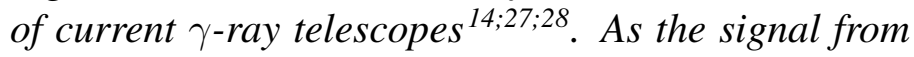
${ }^{56}$ Co $\gamma$-rays is split into temporal bins, statistical precision is compromised (blue: 11 time bins; red: 4 time bins; $1 D$ models are shown as dashed/dotted/solid curves). Non-spherical effects may be more important than $1 D$ models indicate, based on the measurements of radiation processed by the supernova envelope. A future $\gamma$-ray telescope will measure many SNIa with a significantly improved precision that complements UV/optical/IR measurements.

ment in space of a new and significantly better $\gamma$-ray telescope.

A line sensitivity 1-2 orders of magnitude better than previous generation instruments $\left(\simeq 1 \times 10^{-7}\right.$ ph $\mathrm{cm}^{-2} \mathrm{~s}^{-1}$ for broad lines over the $0.05-3.0 \mathrm{MeV}$ range) and a large field of view ( $\gtrsim 2.5 \mathrm{sr}$ ) will, for the first time, unlock systematic time-domain SNIa population studies. High-precision measurements of the ${ }^{56} \mathrm{Ni} \gamma$-ray light curve (see Fig. 1) can check and improve the optical/IR derived luminositywidth relation. Measuring SNIa $\gamma$-ray light curves beginning within 1 day of the shock breaching the stellar surface and extending to 100 days, coupled with resolving key radionuclide line features (not just $\left.{ }^{56} \mathrm{Co}\right)^{\sqrt{99}}$ in the spectra every 5 days, of 10 -100 events/yr out to distance of $\leq 100 \mathrm{Mpc}$, will provide a significant improvement in our understanding of the SNIa progenitor system(s) and explosion mechanism(s). Time-domain characterization of the emergent SNIa $\gamma$-rays will facilitate the extraction of physical parameters such as explosion energy, total mass, spatial distribution of nickel masses 29 , and ultimately lead to the astrophysical modeling and understanding of progenitors and explosion mechanisms. The relevant $\gamma$-ray light curves can be extracted from integrated MeV spectra (bolometric), resolved nuclear lines, or physics-motivated energy bands. Detection of several SNIa will distinguish between the models; population studies involving $\gtrsim 100$ SNIa will be transformational.

An MeV $\gamma$-ray mission will also act as an early time monitor/alert system of SNIa in dusty environments like the Milky Way plane and nearby starburst regions. Dust obscuration could delay 
optical/IR identification of a SNIa for $\gtrsim 2$ weeks, but a $\gamma$-ray line detection will be a unique means of identifying SNIa as early as $\lesssim 10$ days, especially if surface ${ }^{56} \mathrm{Ni}$ exists as suggested by SN2014J $\gamma$-ray observations ${ }^{92 / 27 \mid 52}$ (see Fig. 1), increasing early detection rates and maximizing science returns.

A new $\gamma$-ray radionuclide mission is timely: the current INTEGRAL and NUSTAR missions are in their late phases. A new $\gamma$-ray radionuclide mission improved by technological advances made in the past decade will provide unique data of significant interest across a range of topics to the broad astronomical community, complementary to the multi-messenger data also provided by JWST, LSST, ALMA, TESS, fermi, TMT, GMT, SKA, Gaia, IceCube, CTA, JUNO, FRIB, ATLAS and LIGO.

Cataclysmic variables are semi-detached binary systems consisting of a white dwarf accreting from a low mass stellar companion $58|38| 103|54| 84$. They are progenitors for nova events, with classical novae being the most optically luminous subclass ${ }^{53 \mid 109] 78}$. Some classes of novae may be the progenitors of a population of $\mathrm{SNIa}^{[45|102| 108|109| 101}$. Two types of $\mathrm{MeV} \gamma$-ray emission are expected from novae: prompt emission from $e^{-} e^{+}$annihilation with the $e^{+}$originating from ${ }^{13} \mathrm{~N}$ and ${ }^{18} \mathrm{~F}$, and a longer-lasting emission from ${ }^{7} \mathrm{Be}$ and ${ }^{22} \mathrm{Na}$ decays ${ }^{62139}$. The prompt emission has a $\lesssim 1$ day duration and appears $\simeq 1-2$ weeks before optical maximum, and the longer-lasting emission persists for $\simeq 0.1-3$ yr. Recent UV detections of a few novae suggest the ${ }^{7} \mathrm{Be}$ ejecta mass is larger than current $1 \mathrm{D}$ models produce ${ }^{110[111 \mid 76}$. A next-generation $\gamma$-ray mission as described above will allow, for the first time, systematic time-domain studies of novae populations. Such explorations will address key uncertainties about mixing between the accreted matter and the white dwarf, the conversion of radioactivity into optical emission, and the contribution of novae to galactic enrichment. In addition, measurements at facilities such as ARIEL, ATLAS, and FRIB and stable beam facilities will approach a complete set of reaction rates for classical novae ${ }^{77}$ on a similar timeline for a next-generation gamma-ray mission.

Other cosmic explosions such as core-

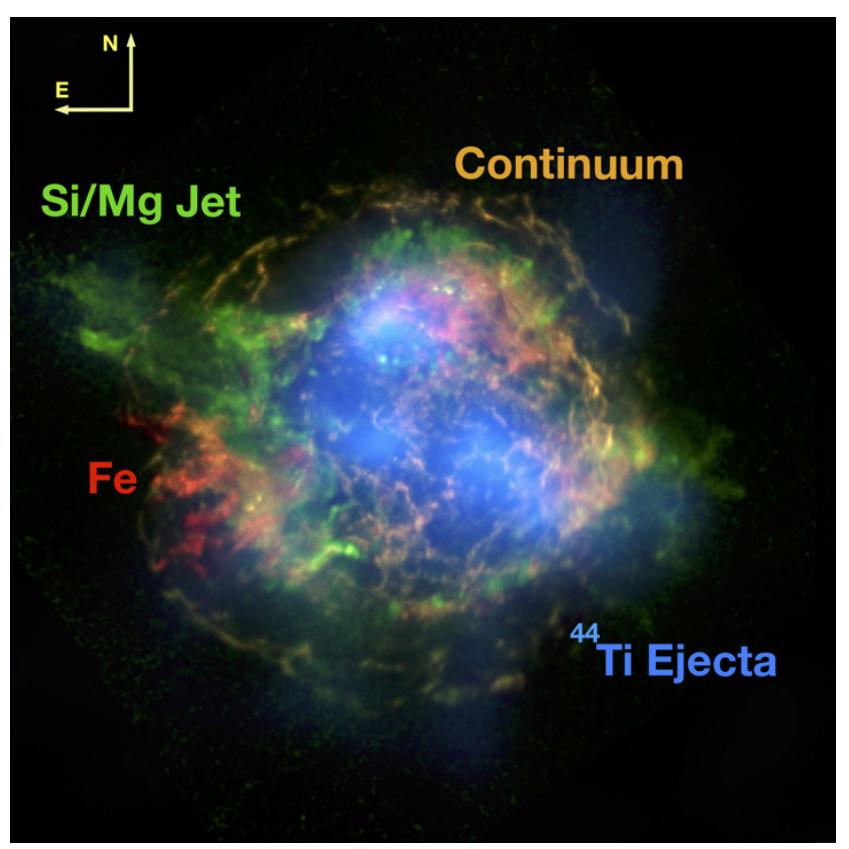

Figure 2: $3 D$ distribution of Cas A ejecta. NuSTAR ${ }^{44}$ Ti in blue, Chandra continuum in gold, $\mathrm{Si} / \mathrm{Mg}$ band in green, $X$-ray emitting iron in red ${ }^{43}$. collapse supernovae (CCSN), pair instability supernovae, neutron star mergers, fast radio bursts, and gamma-ray bursts are also expected to exhibit key signatures about their interior workings that can be observed with a modern $\gamma$-ray telescope. For example, the spatial distribution of elements in young supernova remnants directly probes the dynamics and asymmetries traced by, or produced by, explosive nucleosynthesis ${ }^{80 \mid 128}$.

One crucial diagnostic in young remnants is the relative production of ${ }^{44} \mathrm{Ti},{ }^{56} \mathrm{Ni}$, and ${ }^{28} \mathrm{Si}$. These have indirectly been observed in X-rays from atomic transitions, and $\gamma$-rays from radioactive decay have shown how this can be misleading about where the newly-formed elements actually reside (see Fig. 2). The physical processes that produce these isotopes in CCSN depend on the local conditions of the shock during explosive nucleosynthesis $115|116| 131|119| 68 \mid 13$. The isotope ${ }^{44} \mathrm{Ti}\left(\tau_{1 / 2} \simeq 60 \mathrm{yr}{ }^{4433}\right)$ offers a key diagnostic of the explosion mechanism ${ }^{94 / 50 \mid 41 / 9] 42 / 43}$ because its synthesis is the most sensitive to the local condi- 
tions. For example, Cas A was an excellent target for current $\gamma$-ray instruments because it is young $(\simeq 340 \mathrm{yr})$ and nearby $(\simeq 3.4 \mathrm{kpc})$. Its ejecta has been monitored for decades at X-ray/optical/IR wavelengths, which are now understood to only provide complementary insight into the dynamics

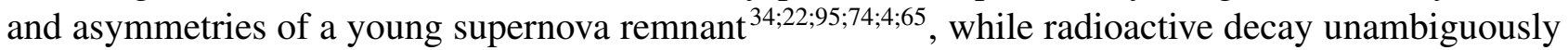
traces the flow and dynamics of new ejecta.

To date, only Cas A and SN 1987A have been used to place constraints on CCSN progenitors and explosion mechanisms $114|104| 60|100| 121$. A new $\mathrm{MeV} \gamma$-ray mission with the characteristics described above will detect $\simeq 8$ young supernova remnants in the Milky Way ${ }^{30}$ and provide a precise abundance measurement of ${ }^{44} \mathrm{Ti}$ in the remnant of SN $1987 \mathrm{~A}^{41|9| 121}$. New measurements of a few CCSN in their ${ }^{44} \mathrm{Ti}$ light will add to our knowledge; population studies with a four-times larger sample size to determine the variation in ${ }^{44} \mathrm{Ti}$ yields from CCSN will be groundbreaking.

\section{Tracing Chemical Evolution}

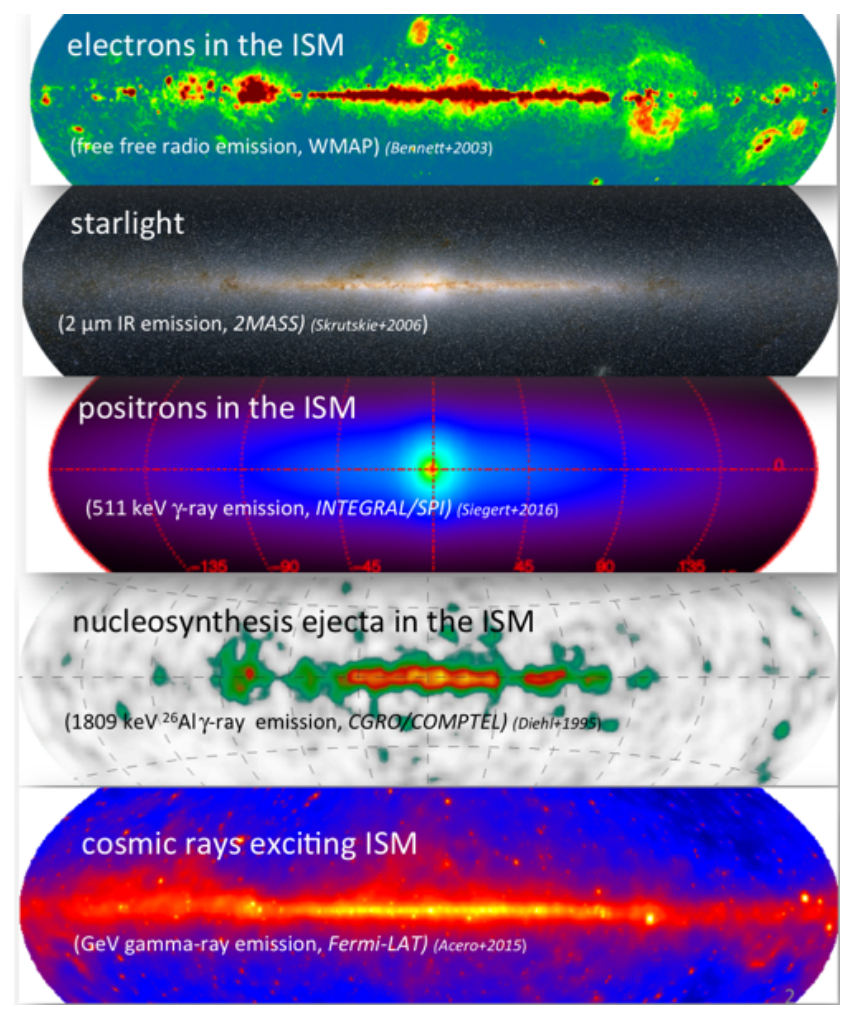

Figure 3: Deciphering the Milky Way. A modern MeV $\gamma$-ray instrument will help solve how newly created elements are produced, transported, mixed, and distributed.
The star-gas-star cycle operating in the evolution of galaxies includes at least four phases where $\mathrm{MeV} \gamma$-ray astronomy provides unique and direct diagnostics of cosmic explosions and chemical evolution. (1) The ejected yields of radionuclides by stars and explosive nucleosynthesis events tell us about the otherwise hidden conditions of nuclear fusion reactions in these sites. (2) The flow of stellar ejecta into the ambient gas (i.e., mixing in chemical evolution) is directly traced by radionuclides over their radioactive lifetimes, which is possible because the $\gamma$-ray emitting nuclear decays are independent of the thermodynamics or composition of the ambient gas. (3) Positrons emitted by radioactive decays, visible through their annihilation $\gamma$-rays, tell us about the nucleosynthesis in individual events and the structure and dynamics of the Galaxy. (4) Nuclear de-excitation $\gamma$-rays caused by cosmic ray collisions with the ambient gas provide the most direct measurements of the cosmic ray flux at $\mathrm{MeV}$ energies and illuminate otherwise invisible fullyionized gas (e.g., the hot ISM and IGM). These four items are the science drivers for a new $\gamma$-ray mission in the 2020s.

Because their lifetimes are long (ten times longer than observables at any other wavelength) compared to the interval between massive star supernovae, yet abundant enough to yield detectable emission when they decay, the radionuclides ${ }^{26} \mathrm{Al}\left(\tau_{1 / 2} \simeq 7.3 \times 10^{5} \mathrm{yr}{ }^{82 / 117}\right)$ and ${ }^{60} \mathrm{Fe}\left(\tau_{1 / 2} \simeq 2.6 \times 10^{6}\right.$ $\mathrm{yr}^{97 / 85}$ ) are valuable tools of $\gamma$-ray astronomy for advancing our global understanding of massive stars and their supernova explosions. This includes the complex late phases of stellar evolution $130|11| 20|19| 10|79| 83$, actions of neutrinos ${ }^{36|86| 87}$ and supernova shockwaves ${ }^{8 / 33}$, and how ejecta of new elements from these 
sources are spread in galaxies 571123 . The clock inherent to emission from radioactivity again helps here, as in the case of Cas A above, to illuminate otherwise invisible, tenuous gas flows. The shortlived radionuclides ${ }^{26} \mathrm{Al},{ }^{60} \mathrm{Fe},{ }^{53} \mathrm{Mn}$, and ${ }^{182} \mathrm{Hf}$ present in the early Solar System play a pivotal role in constraining its formation and chronology. Furthermore, ${ }^{26} \mathrm{Al}$ is the major heating source for thermal and volatile evolution of small planetesimals in the early Solar System ${ }^{122 \mid 61777112 / 66}$.

Current $\gamma$-ray instruments measure the diffuse emission from ${ }^{26} \mathrm{Al}$ and ${ }^{60} \mathrm{Fe}$ decays in the inner portions of the Milky Way Galaxy ${ }^{93110725 \mid 125}$ (see Fig. 3), and the bulk dynamics of ${ }^{26} \mathrm{Al}$ through Doppler shifts and broadening of the $\gamma$-ray line for 3-4 massive-star groups / OB associations $\frac{7026159}{}$. This provides a key test for models of stellar feedback in galaxies, including massive-star winds, supernova explosion energy, and abundance mixing physics. A new $\gamma$-ray instrument with a line sensitivity 1-2 orders of magnitude better than previous instruments $\left(\simeq 1 \times 10^{-7} \mathrm{ph} \mathrm{cm}^{-2} \mathrm{~s}^{-1}\right.$ for broad lines over $0.05-3.0 \mathrm{MeV}$ ), angular resolution of $1-2^{\circ}$, and energy resolution of $0.1 \%$ (to differentiate the emission lines from specific $\mathrm{OB}$ associations against the diffuse radioactive afterglow of stellar activity), will increase the number of $\gamma$-ray observed OB associations by an order of magnitude to $25-35$ based on observed distances to OB associations ${ }^{7026673}$.

Another signal addressed by the same new $\gamma$-ray telescope is positron annihilation and its characteristic $\gamma$-ray spectrum, including a line at $511 \mathrm{keV}$. Current telescopes have established a morphology of our Galaxy's annihilation $\gamma$-rays peaking in the inner Galaxy ${ }^{56 / 126105}$, while most candidate sources reside in the Galaxy's disk. Solving this puzzle includes re-examining cosmic rays, supernovae ${ }^{21}$, pulsars, microquasars, the Fermi bubbles, neutron star mergers ${ }^{37}$, and possibly dark matter emission.

\section{When Opportunity Knocks}

A new $\mathrm{MeV} \gamma$-ray observatory offers considerable serendipitous science for uncommon or surprising events such as a nearby CCSN, neutron star merger, or fast radio burst ${ }^{23}$. Their detection in $\gamma$-rays could entirely restructure our understanding of both the transient itself and its implications for astrophysics as a whole. For example, a detector with a line sensitivity 50 times greater than current instruments will detect 7 radioactive isotopes $\left({ }^{48} \mathrm{Cr},{ }^{48} \mathrm{~V},{ }^{52} \mathrm{Mn},{ }^{56-57} \mathrm{Co},{ }^{56-57} \mathrm{Ni}\right)$ from a CCSN occurring within $1 \mathrm{Mpc}$ and 7 more $\left({ }^{43} \mathrm{~K},{ }^{44} \mathrm{Ti},{ }^{44} \mathrm{Sc},{ }^{47} \mathrm{Sc},{ }^{47} \mathrm{Ca},{ }^{51} \mathrm{Cr},{ }^{59} \mathrm{Fe}\right)$ if within $50 \mathrm{kpc}$. These radionuclides provide a unique and powerful probe of the explosion of massive stars ${ }^{81 / 24}$. Similarly, $\gamma$-rays from the radionuclides produced during the r-process ${ }^{18}$ in a neutron star merger such as GW170817 ${ }^{[12]}$ would be detectable at 3-10 Mpc $c^{48}$. Exact yields from GW170817 are difficult to determine from optical/IR measurements alone, and it is not settled that GW170817 produced the heavy r-process elements ${ }^{6311846147}$. A sufficiently strong $\gamma$-ray signal, coupled with a set of multi-messenger signals, could distinguish between light and heavy r-process production to possibly cement neutron star mergers as the dominant r-process site.

\section{Imagining the Future}

The time is ripe for the astronomy community to strongly advocate for a new $\mathrm{MeV} \gamma$-ray mission to be operational in the 2020s. Such a mission will be based on advanced space-proven detector technology with unprecedented line sensitivity, angular and energy resolution, sky coverage, polarimetric capability, and trigger/alert capability for, and in conjunction with, other multi-messenger instruments. Potential missions include $A M E G O^{[5]}, C C^{[55]}, e-A s t r o G A M^{[6}, E T C C^{[113}, H E X-P^{[67}$, and $L O X^{[5]}$. A new $\mathrm{MeV} \gamma$-ray mission will open unique windows on the Universe by making pioneering observations of cosmic explosions and the flow of their newly created elements into Galactic ecosystems. 


\section{References}

[1] Abbott, B. P., Abbott, R., Abbott, T. D., et al. 2017, ApJL, 848, L13, doi: $10.3847 /$ 2041-8213/aa920c

[2] —. 2017, ApJL, 850, L40, doi: $10.3847 /$ 2041-8213/aa93fc

[3] Ahmad, I., Greene, J. P., Moore, E. F., et al. 2006, Phys. Rev. C, 74, 065803, doi: 10 . 1103 /PhysRevC. 74.065803

[4] Alarie, A., Bilodeau, A., \& Drissen, L. 2014, MNRAS, 441, 2996, doi:10.1093/ mnras/stu 774

[5] AMEGO. 2018, https://asd.gsfc. nasa.gov/amego/

[6] Angelis, A. D., Tatischeff, V., Grenier, I., et al. 2018, Journal of High Energy Astrophysics, doi: https://doi.org/10. $1016 / j$.jheap.2018.07.001

[7] Arcones, A., Bardayan, D. W., Beers, T. C., et al. 2017, Progress in Particle and Nuclear Physics, 94, 1, doi: $10.1016 / j \cdot p p n p$. 2016.12 .003

[8] Blondin, J. M., Mezzacappa, A., \& DeMarino, C. 2003, ApJ, 584, 971

[9] Boggs, S. E., Harrison, F. A., Miyasaka, H., et al. 2015, Science, 348, 670, doi: 10 . $1126 /$ science.aaa2259

[10] Bruenn, S. W., Lentz, E. J., Hix, W. R., et al. 2016, ApJ, 818, 123, doi: $10.3847 /$ $0004-637 \mathrm{X} / 818 / 2 / 123$

[11] Burrows, A., Dolence, J. C., \& Murphy, J. W. 2012, ApJ, 759, 5, doi: $10.1088 /$ $0004-637 \mathrm{X} / 759 / 1 / 5$

[12] Chevalier, R. A. 1976, Nature, 260, 689 doi: $10.1038 / 260689 \mathrm{a} 0$

[13] Chieffi, A., \& Limongi, M. 2017, ApJ, 836, 79, doi: $10.3847 / 1538-4357 /$ $836 / 1 / 79$

[14] Churazov, E., Sunyaev, R., Isern, J., et al. 2014, Nature, 512, 406, doi: $10.1038 /$ nature13672

[15] Colgate, S. A. 1979, ApJ, 232, 404, doi: $10.1086 / 157300$

[16] Colgate, S. A., \& McKee, C. 1969, ApJ, 157, 623, doi: $10.1086 / 150102$

[17] Côté, B., O'Shea, B. W., Ritter, C., Herwig, F., \& Venn, K. A. 2017, ApJ, 835, 128, doi: $10.3847 / 1538-4357 / 835 / 2 / 128$

[18] Côté, B., Fryer, C. L., Belczynski, K., et al. 2018, ApJ, 855, 99

[19] Couch, S. M., Chatzopoulos, E., Arnett, W. D., \& Timmes, F. X. 2015, ApJL, 808, L21, doi: $10.1088 / 2041-8205 / 808 /$ $1 / \mathrm{L} 21$

[20] Couch, S. M., \& Ott, C. D. 2013, ApJL, 778, L7, doi: $10.1088 / 2041-8205 /$ $778 / 1 / \mathrm{L} 7$

[21] Crocker, R. M., Ruiter, A. J., Seitenzahl, I. R., et al. 2017, Nature Astronomy, 1, 0135, doi: $10.1038 / s 41550-017-0135$

[22] DeLaney, T., Rudnick, L., Stage, M. D. et al. 2010, ApJ, 725, 2038, doi: $10.1088 /$
0004-637X/725/2/2038

[23] DeLaunay, J. J., Fox, D. B., Murase, K., et al. 2016, ApJ, 832, L1, doi: $10.3847 /$ 2041-8205/832/1/L1

[24] Diehl, R., Hartmann, D. H., \& Prantzos, N. 2018, Astronomy with Radioactivities (Springer: Berlin), doi: $10.1007 /$ 978-3-319-91929-4

[25] Diehl, R., Halloin, H., Kretschmer, K., et al. 2006, A.\&A, 449, 1025, doi: 10 . 1051/0004-6361:20054301

[26] Diehl, R., Lang, M. G., Martin, P., et al. 2010, A.\&A, 522, A51, doi: $10.1051 /$ 0004-6361/201014302

[27] Diehl, R., Siegert, T., Hillebrandt, W., et al. 2014, Science, 345, 1162, doi: $10.1126 /$ science.1254738

[28] -. 2015, A.\&A, 574, A72, doi: 10 . 1051/0004-6361/201424991

[29] Dong, S., Katz, B., Kushnir, D., \& Prieto, J. L. 2015, MNRAS, 454, L61, doi: 10 . $1093 / \mathrm{mn} r a s l / s l v 129$

[30] Dufour, F., \& Kaspi, V. M. 2013, ApJ, 775, 52, doi: $10.1088 / 0004-637 \mathrm{X} / 775 / 1 /$ 52

[31] Edmon, P. P., Kang, H., Jones, T. W., \& Ma, R. 2011, MNRAS, 414, 3521, doi: 10 . 1111/j.1365-2966.2011.18652.x

[32] Fang, X., Thompson, T. A., \& Hirata C. M. 2018, MNRAS, 476, 4234, doi: 10 . $1093 / \mathrm{mnras} / \mathrm{sty} 472$

[33] Fernández, R. 2015, MNRAS, 452, 2071, doi: $10.1093 / \mathrm{mnras} / \mathrm{stv} 1463$

[34] Fesen, R. A. 2001, ApJS, 133, 161, doi: $10.1086 / 319181$

[35] Foley, R. J., Pan, Y.-C., Brown, P., et al. 2016, MNRAS, 461, 1308, doi: $10.1093 /$ mnras/stw1440

[36] Fröhlich, C., Martínez-Pinedo, G. Liebendörfer, M., et al. 2006, Physical Review Letters, 96, 142502, doi:10.1103/ PhysRevLett.96.142502

[37] Fuller, G. M., Kusenko, A., Radice, D., \& Takhistov, V. 2018, arXiv e-prints, arXiv:1811.00133. https://arxiv. org/abs/1811.00133

[38] Giovannelli, F. 2008, Chinese Journal of Astronomy and Astrophysics Supplement, 8, 237

[39] Gomez-Gomar, J., Hernanz, M., Jose, J., \& Isern, J. 1998, MNRAS, 296, 913, doi: 10. $1046 / j .1365-8711.1998 .01421 . x$

[40] Graur, O., Rodney, S. A., Maoz, D., et al. 2014, ApJ, 783, 28, doi: $10.1088 /$ $0004-637 \mathrm{X} / 783 / 1 / 28$

[41] Grebenev, S. A., Lutovinov, A. A., Tsygankov, S. S., \& Winkler, C. 2012, Nature, 490, 373, doi: $10.1038 /$ nature11473

[42] Grefenstette, B. W., Harrison, F. A., Boggs, S. E., et al. 2014, Nature, 506, 339, doi: $10.1038 /$ nature12997

[43] Grefenstette, B. W., Fryer, C. L., Harrison, F. A., et al. 2017, ApJ, 834, 19, doi: 10 .
3847/1538-4357/834/1/19

[44] Hashimoto, T., Nakai, K., Wakasaya, Y., et al. 2001, Nuclear Physics A, 686, 591, doi: $10.1016 / \mathrm{S} 0375-9474(00)$ $00566-2$

[45] Hernanz, M., \& José, J. 2008, New Astron. Rev., 52, 386, doi: $10.1016 / j . n e w a r$. 2008.06 .017

[46] Holmbeck, E. M., Surman, R., Sprouse, T. M., et al. 2018, ArXiv e-prints, arXiv:1807.06662. https: //arxiv.org/abs/1807.06662

[47] Horowitz, C. J., Arcones, A., Côté, B., et al. 2018, ArXiv eprints, arXiv:1805.04637. https: //arxiv.org/abs/1805.04637

[48] Hotokezaka, K., Wanajo, S., Tanaka, M., et al. 2016, MNRAS, 459, 35, doi: 10 . $1093 / \mathrm{mnras} / \mathrm{stw} 404$

[49] Howell, D. A. 2011, Nature Communications, 2, 350, doi: $10.1038 /$ ncomms 1344

[50] Hwang, U., \& Laming, J. M. 2012, ApJ, 746, 130, doi: $10.1088 / 0004-637 \mathrm{X} /$ $746 / 2 / 130$

[51] Iben, Jr., I., \& Tutukov, A. V. 1984, ApJS, 54,335

[52] Isern, J., Jean, P., Bravo, E., et al. 2016, A.\&A, 588, A67, doi: $10.1051 /$ 0004-6361/201526941

[53] José, J., \& Hernanz, M. 2007, Journal of Physics G Nuclear Physics, 34, 431, doi: 10 . 1088/0954-3899/34/12/R01

[54] Ju, W., Stone, J. M., \& Zhu, Z. 2016, ApJ, 823, 81, doi: $10.3847 / 0004-637 \mathrm{X} /$ $823 / 2 / 81$

[55] Kierans, C. A., Boggs, S. E., Chiu, J.L., et al. 2017, ArXiv e-prints. https: //arxiv.org/abs/1701.05558

[56] Knödlseder, J., Jean, P., Lonjou, V., et al. 2005, A.\&A, 441, 513, doi: $10.1051 /$ 0004-6361:20042063

[57] Kobayashi, C., \& Nakasato, N. 2011, ApJ, 729, 16, doi: $10.1088 / 0004-637 \mathrm{X} /$ $729 / 1 / 16$

[58] Kraft, R. P. 1963, in Advances in Astronomy and Astrophysics, Vol. 2, Advances in Astronomy and Astrophysics, ed. Z. Kopal (Elsevier), 43 85. http://www.sciencedirect. com/science/article/pii/ B9781483199207500064

[59] Kretschmer, K., Diehl, R., Krause, M., et al. 2013, A.\&A, 559, A99, doi: $10.1051 /$ 0004-6361/201322563

[60] Larsson, J., Fransson, C., Östlin, G., et al. 2011, Nature, 474, 484, doi: $10.1038 /$ nature10090

[61] Lee, T., Papanastassiou, D. A., \& Wasserburg, G. J. 1976, Geophys. Res. Lett., 3, 109, doi: $10.1029 /$ GL003i002p00109

[62] Leising, M. D., \& Clayton, D. D. 1987, ApJ, 323, 159, doi: $10.1086 / 165816$

[63] Lippuner, J., Fernández, R., Roberts, L. F., 
et al. 2017, MNRAS, 472, 904, doi: 10 . $1093 / \mathrm{mnras} / \mathrm{st} \times 1987$

[64] Loewenstein, M. 2006, ApJ, 648, 230, doi: $10.1086 / 505648$

[65] Lopez, L. A., \& Fesen, R. A. 2018, Space Sci. Rev., 214, 44, doi: $10.1007 /$ S11214-018-0481-x

[66] Lugaro, M., Ott, U., \& Kereszturi, Á. 2018, Progress in Particle and Nuclear Physics, 102, 1, doi: $10.1016 / j \cdot$ ppnp.2018. 05.002

[67] Madsen, K. K., Harrison, F., Broadway, D., et al. 2018, in Society of Photo-Optical Instrumentation Engineers (SPIE) Conference Series, Vol. 10699, Space Telescopes and Instrumentation 2018: Ultraviolet to Gamma Ray, 106996M

[68] Magkotsios, G., Timmes, F. X., Hungerford, A. L., et al. 2010, ApJS, 191, 66, doi: 10.1088/0067-0049/191/1/66

[69] Maoz, D., Mannucci, F., \& Nelemans, G. 2014, Ann. Rev. Astron.\& Astrophys. , 52, 107, doi: $10.1146 /$ annurev-astro-082812-141031

[70] Martin, P., Knödlseder, J., Diehl, R., \& Meynet, G. 2009, A.\&A, 506, 703, doi: 10 . 1051/0004-6361/200912178

[71] Martizzi, D., Faucher-Giguère, C.-A., \& Quataert, E. 2015, MNRAS, 450, 504, doi: $10.1093 / \mathrm{mnras} / \mathrm{stv} 562$

[72] Matteucci, F., \& Greggio, L. 1986, A.\&A, 154, 279

[73] Mel'nik, A. M., \& Dambis, A. K. 2017, MNRAS, 472, 3887, doi: $10.1093 /$ mnras/stx2225

[74] Milisavljevic, D., \& Fesen, R. A. 2013, ApJ, 772, 134, doi: $10.1088 /$ 0004-637X/772/2/134

[75] Miller, R. S., Ajello, M., Beacom, J. F., et al. 2018, in Deep Space Gateway Concept Science Workshop, Vol. 2063, 3094

[76] Molaro, P., Izzo, L., Mason, E., Bonifacio, P., \& Della Valle, M. 2016, MNRAS, 463, L117, doi:10.1093/mnrasl/slw169

[77] Mostefaoui, S., Lugmair, G. W., \& Hoppe, P. 2005, ApJ, 625, 271, doi: $10.1086 /$ 429555

[78] Mróz, P., Udalski, A., Pietrukowicz, P., et al. 2016, Nature, 537, 649, doi: 10 . 1038/nature19066

[79] Müller, B., Melson, T., Heger, A., \& Janka, H.-T. 2017, MNRAS, 472, 491, doi: 10 . 1093/mnras/stx1962

[80] Nagataki, S., Hashimoto, M.-A., Sato, K., Yamada, S., \& Mochizuki, Y. S. 1998, ApJL, 492, L45+

[81] Nomoto, K., Tominaga, N., Umeda, H., Kobayashi, C., \& Maeda, K. 2006, Nuclear Physics A, 777, 424, doi: $10.1016 / j$. nuclphysa.2006.05.008

[82] Norris, T. L., Gancarz, A. J., Rokop, D. J., \& Thomas, K. W. 1983, in Lunar and Planetary Science Conference Proceedings,
Vol. 14, Lunar and Planetary Science Conference Proceedings, ed. W. V. Boynton \& G. Schubert, B331-B333

[83] O'Connor, E., \& Couch, S. 2018, ArXiv e-prints. https://arxiv.org/abs/ 1807.07579

[84] Oliveira, A. S., Rodrigues, C. V., Cieslinski, D., et al. 2017, AJ, 153, 144, doi: 10 . 3847/1538-3881/aa610d

[85] Ostdiek, K., Anderson, T., Bauder, W., et al. 2015, Nuclear Instruments and Methods in Physics Research B, 361, 638, doi: 10 . 1016/j.nimb.2015.05.033

[86] Patton, K. M., Lunardini, C., \& Farmer, R. J. 2017, ApJ, 840, 2, doi: $10.3847 /$ 1538-4357/aa6ba8

[87] Patton, K. M., Lunardini, C., Farmer, R. J., \& Timmes, F. X. 2017, ApJ, 851, 6, doi: 10 . 3847/1538-4357/aa95c4

[88] Perlmutter, S., Aldering, G., Goldhaber, G., et al. 1999, ApJ, 517, 565

[89] Petrushevska, T., Amanullah, R., Bulla, M., et al. 2017, A.\&A, 603, A136, doi: 10 . 1051/0004-6361/201730989

[90] Phillips, M. M. 1993, ApJL, 413, L105

[91] Phillips, M. M., Lira, P., Suntzeff, N. B., et al. 1999, AJ, 118, 1766

[92] Piro, A. L. 2012, ApJ, 759, 83, doi: 10. 1088/0004-637X/759/2/83

[93] Plüschke, S., Diehl, R., Schönfelder, V., et al. 2001, in ESA Special Publication, Vol. 459, Exploring the Gamma-Ray Universe, ed. A. Gimenez, V. Reglero, \& C. Winkler, $55-58$

[94] Renaud, M., Vink, J., Decourchelle, A., et al. 2006, ApJL, 647, L41, doi: $10.1086 /$ 507300

[95] Rest, A., Foley, R. J., Sinnott, B., et al. 2011, ApJ, 732, 3, doi: $10.1088 /$ $0004-637 X / 732 / 1 / 3$

[96] Riess, A. G., Filippenko, A. V., Challis, P., et al. 1998, AJ, 116, 1009

[97] Rugel, G., Faestermann, T., Knie, K. et al. 2009, Physical Review Letters, 103, 072502, doi: $10.1103 /$ PhysRevLett. 103.072502

[98] Sano, H., Rowell, G., Reynoso, E. M., et al. 2018, ArXiv e-prints. https:// arxiv.org/abs/1805.10647

[99] Seitenzahl, I. R. 2011, Progress in Particle and Nuclear Physics, 66, 329, doi: 10 . 1016/j.ppnp.2011.01.028

[100] Seitenzahl, I. R., Timmes, F. X., \& Magkotsios, G. 2014, ApJ, 792, 10, doi: 10 . 1088/0004-637X/792/1/10

[101] Shafter, A. W. 2017, ApJ, 834, 196, doi: $10.3847 / 1538-4357 / 834 / 2 / 196$

[102] Shafter, A. W., Henze, M., Rector, T. A., et al. 2015, ApJS, 216, 34, doi: $10.1088 /$ $0067-0049 / 216 / 2 / 34$

[103] Shen, K. J., Idan, I., \& Bildsten, L. 2009, ApJ, 705, 693, doi: $10.1088 /$ $0004-637 \mathrm{X} / 705 / 1 / 693$
[104] Shtykovskiy, P. E., Lutovinov, A. A., Gilfanov, M. R., \& Sunyaev, R. A. 2005, Astronomy Letters, 31, 258, doi: $10.1134 / 1$. 1896069

[105] Siegert, T., Diehl, R., Khachatryan, G., et al. 2016, A.\&A, 586, A84, doi:10.1051/ 0004-6361/201527510

[106] Simpson, C. M., Bryan, G. L., Hummels, C., \& Ostriker, J. P. 2015, ApJ, 809, 69, doi: $10.1088 / 0004-637 X / 809 / 1 / 69$

[107] Smith, D. M. 2004, New Astronomy Review, 48,87

[108] Soraisam, M. D., \& Gilfanov, M. 2015, A.\&A, 583, A140, doi: $10.1051 /$ $0004-6361 / 201424118$

[109] Starrfield, S., Iliadis, C., \& Hix, W. R. 2016, PASP, 128, 051001, doi: $10.1088 /$ 1538-3873/128/963/051001

[110] Tajitsu, A., Sadakane, K., Naito, H., Arai, A., \& Aoki, W. 2015, Nature, 518, 381, doi: $10.1038 /$ nature14161

[111] Tajitsu, A., Sadakane, K., Naito, H., et al. 2016, ApJ, 818, 191, doi: $10.3847 /$ 0004-637X/818/2/191

[112] Tang, H., \& Dauphas, N. 2012, Earth and Planetary Science Letters, 359-360, 248, doi: $10.1016 / j . e p s 1.2012 .10 .011$

[113] Tanimori, T., Mizumura, Y., Takada, A., et al. 2017, Scientific Reports, 7, 41511, doi: $10.1038 /$ srep 41511

[114] The, L.-S., Burrows, A., \& Bussard, R. 1990, ApJ, 352, 731, doi: $10.1086 /$ 168575

[115] The, L.-S., Clayton, D. D., Jin, L., \& Meyer, B. S. 1998, ApJ, 504, 500

[116] The, L.-S., Clayton, D. D., Diehl, R., et al. 2006, A.\&A, 450, 1037

[117] Thomas, J. H., Rau, R. L., Skelton, R. T., \& Kavanagh, R. W. 1984, Phys. Rev. C, 30, 385, doi: 10.1103 /PhysRevC.30.385

[118] Thompson, T. A. 2011, ApJ, 741, 82, doi: $10.1088 / 0004-637 X / 741 / 2 / 82$

[119] Timmes, F. X., Woosley, S. E., Hartmann, D. H., \& Hoffman, R. D. 1996, ApJ, 464, 332

[120] Timmes, F. X., Woosley, S. E., \& Weaver, T. A. 1995, ApJS, 98, 617

[121] Tsygankov, S. S., Krivonos, R. A., Lutovinov, A. A., et al. 2016, MNRAS, 458, 3411, doi: $10.1093 / \mathrm{mnras} / \mathrm{stw} 549$

[122] Urey, H. C. 1955, Proceedings of the National Academy of Science, 41, 27, doi: 10 . 1073/pnas.41.1.27

[123] Vincenzo, F., \& Kobayashi, C. 2018, MNRAS, 478, 155, doi:10.1093/mnras/ sty1047

[124] Wang, B., \& Han, Z. 2012, New Astron. Rev., 56, 122, doi: $10.1016 / j$.newar. 2012.04 .001

[125] Wang, W., Harris, M. J., Diehl, R., et al. 2007, A.\&A, 469, 1005

[126] Weidenspointner, G., Skinner, G., Jean, P., et al. 2008, Nature, 451, 159, doi: 10 . 
$1038 /$ nature06490

[127] Whelan, J., \& Iben, I. J. 1973, ApJ, 186, 1007, doi: $10.1086 / 152565$

[128] Wongwathanarat, A., Janka, H.-

T., Müller, E., Pllumbi, E., \&
Wanajo, S. 2017, ApJ, 842, 13, [130] Woosley, S. E., Heger, A., \& Weaver, doi: $10.3847 / 1538-4357 /$ aa72de T. A. 2002, Rev. Mod. Phys., 74, 1015, [129] Wood-Vasey, W. M., Friedman, A. S., doi:10.1103/RevModPhys.74.1015 Bloom, J. S., et al. 2008, ApJ, 689, 377, [131] Young, P. A., Fryer, C. L., Hungerford, doi: $10.1086 / 592374$

A., et al. 2006, ApJ, 640, 891, doi: 10 . $1086 / 500108$ 